\title{
Non-Gaussian Velocity Probability Density Functions: An Altimetric Perspective of the Mediterranean Sea
}

\author{
Jordi IsERn-Fontanet, EMilio García-LAdOna, Jordi Font, AND ANTONio García-Olivares \\ Institut de Ciències del Mar (CSIC), Barcelona, Spain
}

(Manuscript received 6 July 2005, in final form 14 April 2006)

\begin{abstract}
Velocity probability density functions (PDFs) are a key tool to study complex flows and are of great importance to model particle dispersion. The PDFs of geostrophic velocities derived from sea level anomalies maps for the Mediterranean Sea have been computed and analyzed, guided by recent results found in studies of two-dimensional and geostrophic turbulence. At the basin scale results show that the geostrophic velocity PDF derived from SLA maps is non-Gaussian. To understand the origin of this non-Gaussianity, a topological partition of the flow based on the Okubo-Weiss parameter is applied to separate the contribution of coherent vortices from the background field. After such separation the non-Gaussian part of the PDF appears mostly associated with the presence of such structures. Only about $20 \%$ of the vortices identified in the dataset are mainly responsible for this deviation. These vortices, called intense vortices in previous works, are those vortices with values of the amplitude larger than or equal to two standard deviations of the Okubo-Weiss parameter and correspond to vortices with a radius on the order of $40 \mathrm{~km}$.
\end{abstract}

\section{Introduction}

The quasi-bidimensional nature of ocean motions makes two-dimensional turbulence a paradigm for understanding the dynamical properties of ocean flows (Rhines 1979; McWilliams 1984). One of the most remarkable aspects of two-dimensional (2D) turbulent flows is the presence of coherent vortices that emerge under a wide range of initial conditions if forcing and dissipation are sufficiently small. This has led to some widely studied approaches in the past in which such flows are considered as an ensemble of localized vortices that dominate the statistical properties of the flow. The idea of structures with concentrated vorticity as fundamental building blocks of the turbulent flow has been largely exploited in the past to analyze numerical simulations of 2D turbulence, to build conceptual models, and to determine statistical and mixing properties of turbulent flows, scaling laws, and so on (Jiménez 1996; Babiano et al. 1987; McWilliams 1990; Weiss and McWilliams 1993).

Corresponding author address: Jordi Isern-Fontanet, Département d'Océanographic Physique et Spatiale, IFREMER, Centre de Brest Technopôle de Brest-Iroise, BP 70, F-29280 Plouzané, France.

E-mail: jisern@ifremer.fr
A question that recently has received much interest is related to the role of such coherent structures in the dispersion of tracers. Indeed, the presence of vortices induces the anomalous dispersion of passive tracers associated with non-Gaussian statistics of velocity fields (Provenzale 1999; Pasquero et al. 2001). Numerical experiments of decaying turbulence show that the variation of the Reynolds number ( $\mathrm{Re}$ ) modifies the size and separation of the vortices. For small values of Re, the flow is characterized by the presence of large vortices and Gaussian probability density functions (PDFs) of velocity. However, as the Reynolds number increases (and the number of vortices increases, with the vortices becoming smaller), the tails of the velocity PDF smoothly increase. Then, the observed velocity PDFs for large enough Re are non-Gaussian (Bracco et al. 2000a, 2005, manuscript submitted to J. Geophys. Res.). Furthermore, a detailed analysis of the velocity field showed that, although the velocity PDF inside vortices is Gaussian, the velocity field generated by the vorticity of these vortices is not (Bracco et al. 2000a). This suggests that the existence of long tails in the velocity PDF of barotropic turbulence is due to the nonlocal effect of vortices, as was proposed by Jiménez (1996). However, experiments with forced $2 \mathrm{D}$ turbulence have shown that large-scale forcing diminishes the role of coherent 
vortices in the pertinent statistics (Schorghofer and Gille 2002).

Ocean fields also exhibit non-Gaussian velocity PDFs. This has been obtained from the analysis of global satellite altimetry (Llewellyn Smith and Gille 1998; Gille and Llewellyn Smith 2000), drifting buoys (Swenson and Niiler 1996; Bracco et al. 2000b; Maurizi et al. 2004), current meters (LaCasce 2005), and high-resolution numerical simulations of the ocean circulation (Bracco et al. 2003). Such observational evidences lead to the interpretation that the non-Gaussian property may be associated with the presence of coherent vortices that populate the ocean. Nevertheless, it can also be related, as proposed by Llewellyn Smith and Gille (1998), to the large-scale spatial inhomogeneity of the eddy kinetic energy (EKE), which is expected to be higher in regions dominated by the presence of unstable jets. From the analysis of altimeter data it is found that velocity PDFs are predominantly Gaussian for small ocean regions, but in regions dominated by organized flows such as western boundary currents PDFs are closer to an exponential distribution (Llewellyn Smith and Gille 1998). However, in a recent paper, LaCasce (2005) found that Eulerian velocity PDFs computed from current meters in the North Atlantic Ocean were significantly non-Gaussian and statistically indistinguishable from Lagrangian measurements so long as the float data are averaged in bins of appropriate size.

The aim of this work is to analyze to what extent non-Gaussian deviations of velocity PDFs are due to coherent vortices in a semienclosed basin such as the Mediterranean basin. The Mediterranean Sea is dominated by the entrance of freshwater incoming from the Atlantic Ocean through the Strait of Gibraltar. The instability of this inflow and local wind action often generate coherent vortices in several parts of the basin that enhance the mixing of these incoming light waters with the saltier resident waters (e.g., Millot 1999, 2005). Analysis of altimetric maps shows that PDFs of the velocity field at basin scale also appear non-Gaussian, in agreement with previous cited works. To discern to what extent this is due to the presence of mesoscale vortices in the basin we propose to make a partition of the flow between coherent vortices and a background field based on the Okubo-Weiss parameter, following the ideas of Bracco et al. (2000b), and then reanalyze the velocity PDF of both fields.

The paper is structured as follows: section 2 describes the sea level anomaly maps used in this study, the criterion of vortex identification, and the methodology followed to estimate PDFs. Section 3 studies velocity PDFs in different topological domains and presents an explicit separation of the contribution of coherent vortices from other contributions. Section 4 discusses the results and presents conclusions.

\section{Data and methods}

\section{a. Sea level anomalies}

In this study we used sea level anomaly (SLA) maps produced by the Collecte Localisation Satellites (CLS) in Toulouse, France, which combine the signal of the European Remote Sensing Satellite (ERS) and the Ocean Topography Experiment (TOPEX)/Poseidon altimeters. These maps are processed including usual corrections (sea state bias, tides, inverse barometer, etc.) and with improved ERS orbits using TOPEX/Poseidon as a reference (CLS 1996; Le Traon and Ogor 1998). SLA is regularly produced by subtracting a 4-yr mean value (1993-96) and, prior to the analysis, data are lowpass filtered using a $35-\mathrm{km}$ median filter and a Lanczos filter with a cutoff wavelength of $42 \mathrm{~km}$ in order to reduce altimetric noise (Larnicol et al. 1995). The data used here actually span 7 years from October 1992 to October 1999, but with a gap between December 1993 and March 1995 due to the 168-day ERS-1 orbit. SLA maps are finally built every 10 days using an improved space-time objective analysis method, which takes into account long wavelength errors, on a regular grid of $0.2^{\circ} \times 0.2^{\circ}$ (Le Traon et al. 1998; Larnicol et al. 2002) This leaves a total of 213 SLA maps. Data in the Aegean Sea were not considered because of the high density of islands. Velocities are estimated assuming a geostrophic relationship.

\section{b. Vortex extraction}

Vortices are defined, as in previous works (e.g., Pasquero et al. 2001; Isern-Fontanet et al. 2003, 2006), as the simply connected regions with values of the Okubo-Weiss parameter, W (Okubo 1970; Weiss 1991):

$$
W<-0.2 \sigma_{W},
$$

with $\sigma_{W}$ the standard deviation of $W$. This parameter is defined as

$$
W=s_{n}^{2}+s_{s}^{2}-\omega^{2},
$$

where $s_{n}=\partial_{x} u-\partial_{y} v$ and $s_{s}=\partial_{x} v+\partial_{y} u$ are the normal and shear components of strain and $\omega=\partial_{x} v-\partial_{y} u$ is the relative vorticity. Figure 1 (top) shows the spatial distribution of all observed vortex centers for the data analyzed here. After vortices are identified, they are classified in terms of their amplitude (a), as proposed in Isern-Fontanet et al. (2006). Amplitude is defined as 


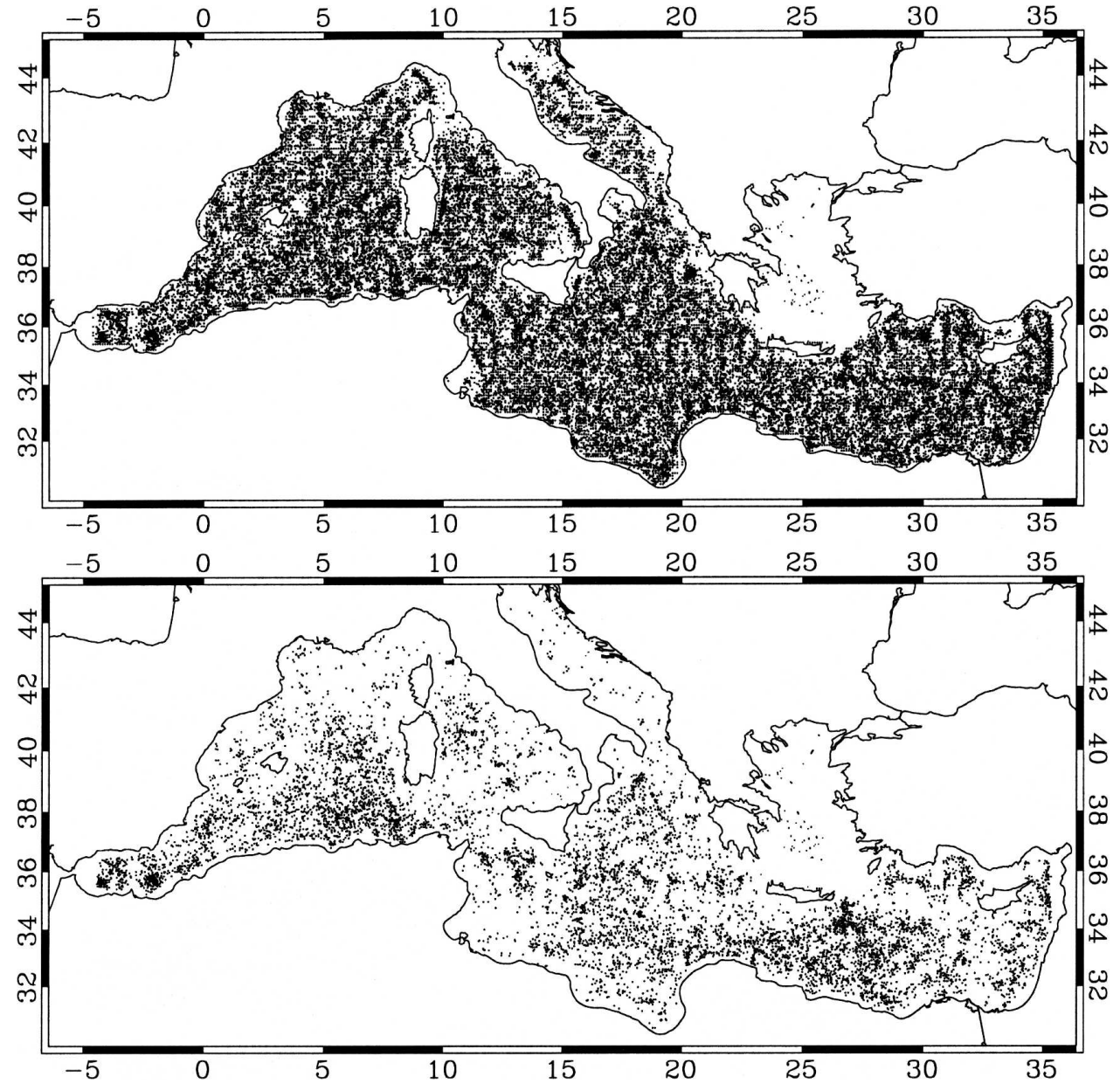

FIG. 1. Distribution of the centers of (top) all vortices and (bottom) intense vortices $\left(a_{0}>2\right)$ identified in the dataset. Adapted from Isern-Fontanet et al. (2006).

the maximum absolute value of the Okubo-Weiss parameter inside the vortex:

$$
a \equiv \max \left(\left|W_{i}\right|\right) \quad i=1, \ldots, N_{\mathrm{gp}},
$$

where $N_{\mathrm{gp}}$ is the number of grid points within the vortex. As is shown by Isern-Fontanet et al. (2006), the kinetic energy of identified vortices is linearly correlated with the amplitude but their spatial scale depends in a nonlinear way on the amplitude. Roughly speaking, vortex size saturates around $a=1.5 \sigma_{W}-2 \sigma_{W}$ with characteristic radius of about $35-40 \mathrm{~km}$. Then, coherent vortices are defined as those vortices with $a>a_{0}$, where $a_{0}$ is a threshold value taken as $a_{0}=2 \sigma_{W}$. In the following, we refer to coherent vortices as intense vortices $(a>$ $\left.2 \sigma_{W}\right)$ and the remaining vortices, those with $a \leq 2 \sigma_{W}$, are referred to as weak vortices. Figure 1 (bottom) shows the spatial distribution of intense or coherent vortices in the Mediterranean Sea. The spatial distribution of vortices over the whole analyzed period is uni- form while the distribution of coherent or intense vortices is rather inhomogeneous. Intense coherent vortices appear more concentrated in some subbasins, while other regions are less populated. Comparisons with some independent datasets have shown that such coherent structures are well correlated and coincide in several cases with in situ sampling, buoys trajectories, and infrared images (Matteoda and Glenn 1996; Puillat et al. 2002; Salas et al. 2002; Isern-Fontanet et al. 2003; Font et al. 2004).

\section{c. Velocity PDF}

Probability density functions of the velocity at basin scale have been estimated by computing the histograms of both components (zonal and meridional) of the geostrophic velocity calculated from the whole time series of SLA maps. First, the mean value of the set has been removed and, then, the velocities have been normalized by the number of samples and divided by the bin width. 
TABLE 1. Number of points $(N)$, variance $\left(\sigma^{2}\right)$, skewness $(s)$, and kurtosis $(k$; its definition includes a -3 term that makes it zero for a Gaussian distribution) of the zonal $(u)$ and meridional $(v)$ components of the velocities defined in the text (see the rightmost column). Velocities labeled $\mathbf{v}$ are the observed velocities or subsets of the observed velocities; velocities labeled $\mathbf{u}$ are velocities obtained by integration of the vorticity $\left(\mathrm{cm} \mathrm{s}^{-1}\right)$, and $\alpha$ is the Kolmogorov-Smirnov statistics.

\begin{tabular}{lrrrrrrrrrr}
\hline \hline & \multicolumn{1}{c}{$N$} & $\sigma_{u}^{2}$ & \multicolumn{1}{c}{$s_{u}$} & $k_{u}$ & $\alpha_{u}$ & $\sigma_{v}^{2}$ & \multicolumn{1}{c}{$s_{v}$} & $k_{v}$ & $\alpha_{v}$ & Section \\
\hline $\mathbf{v}$ & 1149774 & 58.8 & 0.114 & 2.37 & 0.045 & 61.1 & -0.086 & 2.69 & 0.043 & $4 \mathrm{a}$ \\
$\mathbf{v}_{\mathrm{bg}}$ & 879972 & 54.6 & 0.119 & 2.49 & 0.043 & 56.8 & -0.082 & 2.83 & 0.041 \\
$\mathbf{v}_{v}$ & 269802 & 73.0 & 0.028 & 1.89 & 0.036 & 75.3 & -0.055 & 2.16 & 0.035 & $4 \mathrm{a}$ \\
$\mathbf{v}_{w v}$ & 171895 & 38.3 & 0.115 & 0.73 & 0.017 & 38.8 & 0.002 & 0.64 & 0.014 & $4 \mathrm{a}$ \\
$\mathbf{v}_{i v}$ & 97907 & 133.7 & 0.000 & 0.47 & 0.010 & 139.1 & -0.061 & 0.66 & 0.009 & $4 \mathrm{a}$ \\
$\mathbf{u}_{\mathrm{bg}}$ & 1149774 & 12.7 & 0.160 & 0.74 & 0.018 & 15.1 & -0.105 & 0.72 & 0.018 & $4 \mathrm{~b}$ \\
$\mathbf{u}_{v}$ & 1149774 & 33.0 & -0.034 & 2.97 & 0.047 & 38.9 & -0.043 & 3.21 & 0.048 & $4 \mathrm{~b}$ \\
$\mathbf{u}_{w v}$ & 1149774 & 14.9 & -0.001 & 1.14 & 0.025 & 17.5 & -0.097 & 0.99 & 0.025 & $4 \mathrm{c}$ \\
$\mathbf{u}_{i v}$ & 1149774 & 33.5 & -0.091 & 3.65 & 0.066 & 40.3 & 0.045 & 3.77 & 0.062 & $4 \mathrm{c}$ \\
$\mathbf{v}-\mathbf{u}_{i v}$ & 1149774 & 45.8 & 0.157 & 0.68 & 0.020 & 49.7 & -0.097 & 0.63 & 0.017 & $4 \mathrm{c}$ \\
\hline
\end{tabular}

Usually, a reasonably interval of the bin width is between $0.2 \sigma$ and $(0.4-0.5) \sigma$ to have a normalized bias error less than 1\% (Bendat and Piersol 1985; Emery and Thomson 1998). This bin width is a compromise between the reduction of the random error and the suppression of the bias error. Table 1 lists the values of $\sigma$ for all velocity fields considered here. From the whole range of values in the table a bin width of $2 \mathrm{~cm} \mathrm{~s}^{-1}$ has been taken, which is between the lower bound of $0.7 \mathrm{~cm} \mathrm{~s}^{-1}(\approx 0.2 \sigma)$ and a very conservative value of $4.68 \mathrm{~cm} \mathrm{~s}^{-1}(\approx 0.4 \sigma)$.

To quantify the statistical significance of the deviations from a Gaussian distribution the, most widely used test is the Kolmogorov-Smirnov (KS), based on the largest discrepancy between the cumulative density function (CDF) of two PDFs. From a set of observed values $\left\{X_{i}\right\}$, the $\mathrm{KS}$ statistic is defined as

$$
\alpha_{X}=\max \left[\left|F_{n}(X)-F(X)\right|\right],
$$

where $F(X)$ is the observed CDF and $F_{n}(X)$ is the theoretical $\mathrm{CDF}$, in our case a Gaussian with the same variance as the observations. Given this measure of the deviation from a theoretical distribution, it is possible to calculate from an analytical expression the probability of finding a larger value than the observed $[P(\alpha>$ $\left.\alpha_{\text {obs }}\right)$ ] under the assumption that the null hypothesis is true (Press et al. 1994). In our case the null hypothesis is that $\left\{X_{i}\right\}$ are normally distributed. This test was applied to all velocity fields discussed in this paper as well as to subsamples of these fields composed of $10^{4}$ and $2 \times 10^{4}$ randomly picked observations. Results allow one to reject the null hypothesis with $95 \%$ confidence for all situations, except for the zonal component of $\mathbf{v}_{i v}$ (see the next section for its definition) when only $10^{4}$ points are picked $\left[P\left(\alpha>\alpha_{\text {obs }}\right)=12.3 \%\right]$. Nevertheless, this test is more sensitive to deviations in the center of the distribution than in the wings. Then, to reinforce the statistical confidence in the results, we have also applied the Jarque-Bera (JB) test (Jarque and Bera 1981; LaCasce 2005), which, in contrast to the KS test, is more sensitive to deviations in the wings of the distribution. The application of this test to the observed velocities and subsamples shows that the null hypothesis can be rejected in all cases (not shown).

The above estimation of the results' significance has been done by considering that the observed values, or at least a randomly picked subset of observations, were independent. To improve the reliability of significance tests, we have also tried to give a rough estimation of the degrees of freedom of the system. The total number of observations is $10^{6}$ for the whole time series, distributed onto 213 maps separated every 10 days and with about $5 \times 10^{3}$ points per map. Previous altimetric studies suggested that the spatial correlation scale lies between 80 and $100 \mathrm{~km}$ and the temporal correlation scale between 10 and 20 days (Ayoub et al. 1998). Therefore, to construct SLA maps, the temporal scale was chosen to be 15 days and the spatial scale $150 \mathrm{~km}$, which also considers the need to build an isotropic estimation of the SLA field (Larnicol et al. 2002). The number of grid points within a circle of radius equal to $150 \mathrm{~km}$ is approximately 187 . Then, we consider that the number of independent points within a single SLA map is around $5 \times 10^{3} / 187 \simeq 29$. On the other hand, if a temporal correlation scale of 10 were selected, which is consistent with not only Ayoub et al.'s (1998) estimation but also Salas et al. (2001), each map would be considered as an independent realization. However, since this scale has been taken as 15 days for the SLA maps, we consider that there are only $213 / 1.5=142$ independent maps. Therefore, we estimate the number of degrees of freedom as $142 \times 29=4118$. Returning to the above significance tests, if the values of skewness and kurtosis, reported in Table 1, are considered along with our es- 


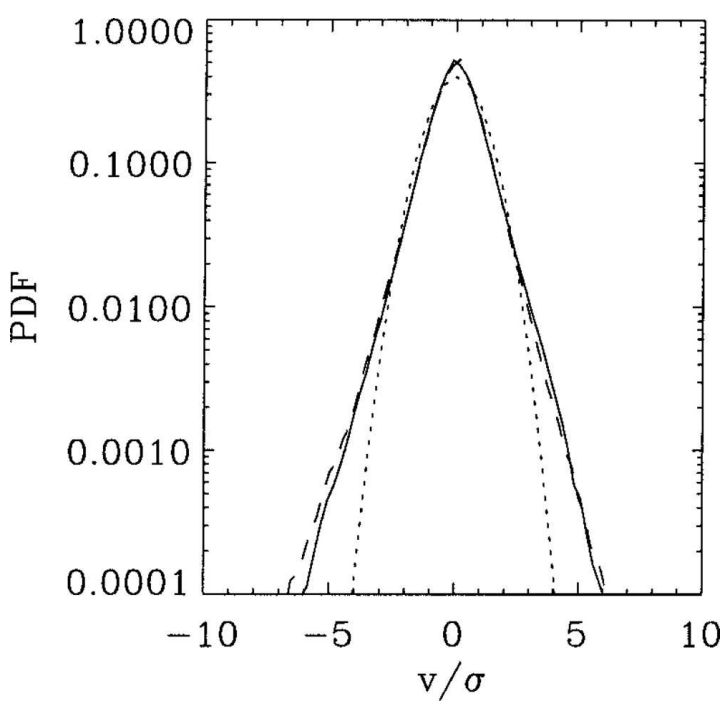

FIG. 2. Velocity PDF for the Mediterranean Sea. The solid line is for the zonal component, the dashed line is for the meridional component, and the dotted line is a Gaussian distribution with $\sigma=1$.

timation of the degrees of freedom, the JB test indicates that the null hypothesis can be rejected in all the situations considered at the $95 \%$ level of confidence (not shown).

\section{Results}

\section{a. Observed PDF}

Figure 2 shows the resulting PDFs of both velocity components, and their moments are listed in the first row of Table 1 . These PDFs are characterized by long tails and a sharp core indicating that its departure from the Gaussian distribution is due to not only energetic events but also a larger contribution of small values. Therefore, the distribution resembles an exponential distribution. This departure from Gaussianity is also evident from the values of kurtosis, which are greater than 0 [the definition of kurtosis includes a -3 term that makes it zero for a Gaussian distribution; Press et al. (1994)].

Since the dynamical characteristics of the flow inside and outside coherent vortices are very different, observed velocities have been split into two subsets. They correspond to two different topological domains of the flow: the background velocity field $\left(\mathbf{v}_{\mathrm{bg}}\right)$, defined as those velocities located in regions with $W \geq-0.2 \sigma_{W}$, and the field inside vortex cores $\left(\mathbf{v}_{\boldsymbol{v}}\right)$, defined as those velocities located in regions with $W<-0.2 \sigma_{W}$. These fields are such that

$$
\mathbf{v}=\mathbf{v}_{\mathrm{bg}} \cup \mathbf{v}_{v} \quad \text { and } \quad \mathbf{v}_{\mathrm{bg}} \cap \mathbf{v}_{v}=\emptyset .
$$
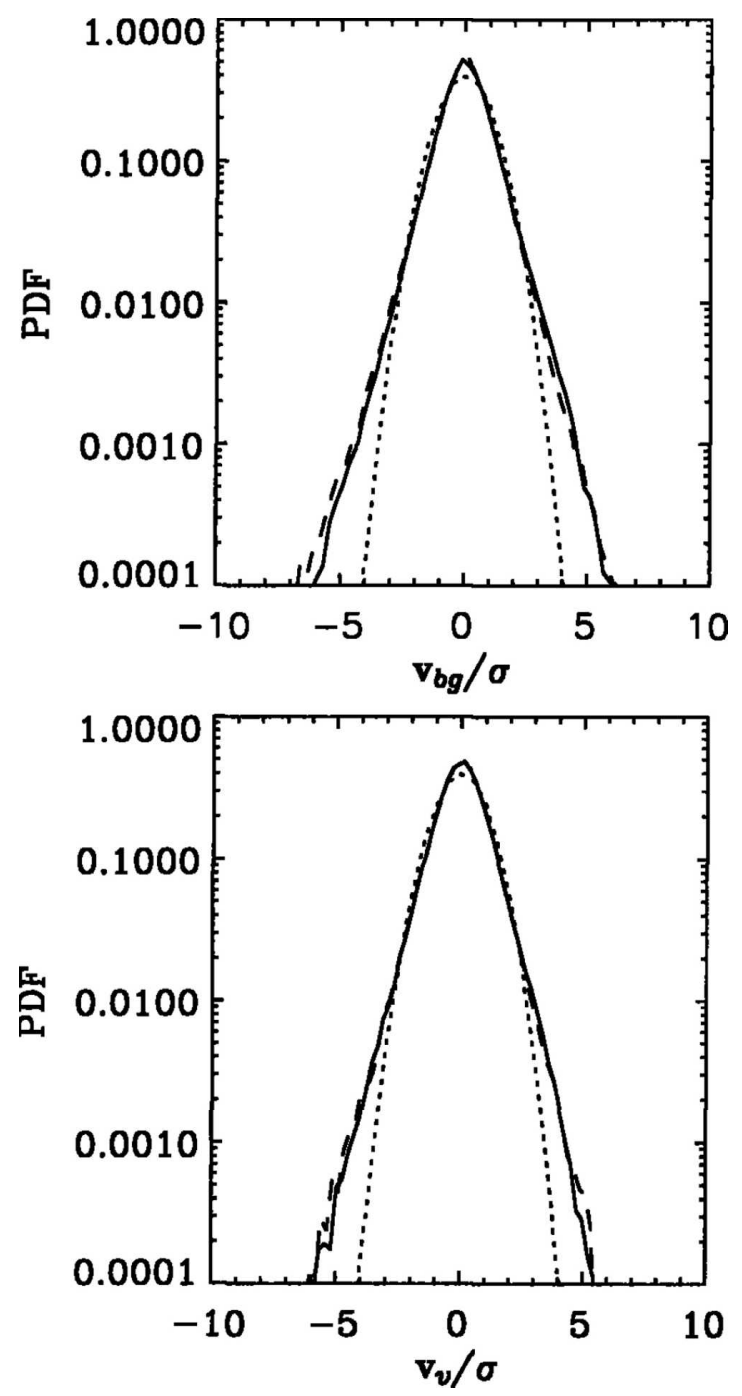

FIG. 3. Velocity PDFs outside of vortices $\left(\mathbf{v}_{\mathrm{bg}}\right)$ and inside vortices $\left(\mathbf{v}_{v}\right)$ for the Mediterranean Sea. The solid line is for the zonal component, the dashed line is for the meridional component, and the dotted line is a Gaussian distribution with $\sigma=1$.

Results (Fig. 3 and Table 1) show that both distributions differ from a Gaussian distribution because of their larger tails and sharper cores. Here $\mathbf{v}_{\mathrm{bg}}$ has higher values of kurtosis and lower energy than $\mathbf{v}_{v}$.

The observed velocity PDFs in SLA maps are nonGaussian when they are observed at basin scale. This is true, even if observations are separated into two subsets with different dynamical properties. It is interesting to compare this result with numerical simulations of two-dimensional turbulence. Bracco et al. (2000a) found that the velocity field inside vortices has a distribution close to a Gaussian. This contrasts with our results. Obviously, the dynamic regime of the Mediterranean Sea (stratified, semienclosed, and forced) is dif- 
ferent from the regimes that they investigated despite the similarities between two-dimensional turbulence and ocean dynamics and, therefore, any comparisons should be done with caution. However, in our opinion, a possible explanation for this discrepancy may be the wide range of energy and sizes associated with ocean vortices observed in altimetric maps. Indeed, if the velocity field inside vortices is split into a contribution due to weak vortices (low energy) and another due to intense vortices (high energy),

$$
\mathbf{v}_{v}=\mathbf{v}_{w v} \cup \mathbf{v}_{i v} \text { and } \quad \mathbf{v}_{w v} \cap \mathbf{v}_{i v}=\emptyset \text {, }
$$

then the shapes of the resulting PDFs for $\mathbf{v}_{w v}$ and $\mathbf{v}_{i v}$ are much closer to a Gaussian than before, although they still have significantly non-Gaussian tails (see Table 1, Fig. 4).

\section{b. Vortex separation}

In the previous section the velocity field was analyzed within different topological domains: background, weak, and intense vortices according to the Okubo-Weiss criterion. However, to separate the contribution of vortices, one needs to take into account that the velocity is an integral of the vorticity. This implies that the background field $\mathbf{v}_{\text {bg }}$ contains contributions from the background distribution of vorticity as well as from the distribution of coherent structure vorticity. Accordingly, we first separate the vorticity field into background and vortex-induced components and then reconstruct the associated velocity field following a similar criterion as that proposed by Bracco et al. (2000a). A velocity field $\mathbf{v}$ with vorticity $\boldsymbol{\omega}$ and divergence $\Delta$ can be decomposed into three contributions (Batchelor 1967):

$$
\mathbf{v}=\mathbf{u}_{1}+\mathbf{u}_{2}+\mathbf{u}_{3},
$$

where $\mathbf{u}_{1}$ is the irrotational velocity field associated with a given rate of expansion $\Delta$ :

$$
\boldsymbol{\nabla} \times \mathbf{u}_{1}=\mathbf{0} \text { and } \boldsymbol{\nabla} \cdot \mathbf{u}_{1}=\Delta,
$$

$\mathbf{u}_{2}$ is the incompressible velocity field associated to a given vorticity distribution:

$$
\boldsymbol{\nabla} \times \mathbf{u}_{2}=\boldsymbol{\omega} \text { and } \boldsymbol{\nabla} \cdot \mathbf{u}_{2}=0,
$$

and $\mathbf{u}_{3}$ is the nondivergent and irrotational flow associated with the boundary conditions:

$$
\boldsymbol{\nabla} \times \mathbf{u}_{3}=\mathbf{0} \text { and } \boldsymbol{\nabla} \cdot \mathbf{u}_{3}=0 .
$$

Vortices are regions of dominance of vorticity. Therefore, since our interest is in the contribution of vortices to the velocity PDF, the decomposition of velocities has been rearranged as
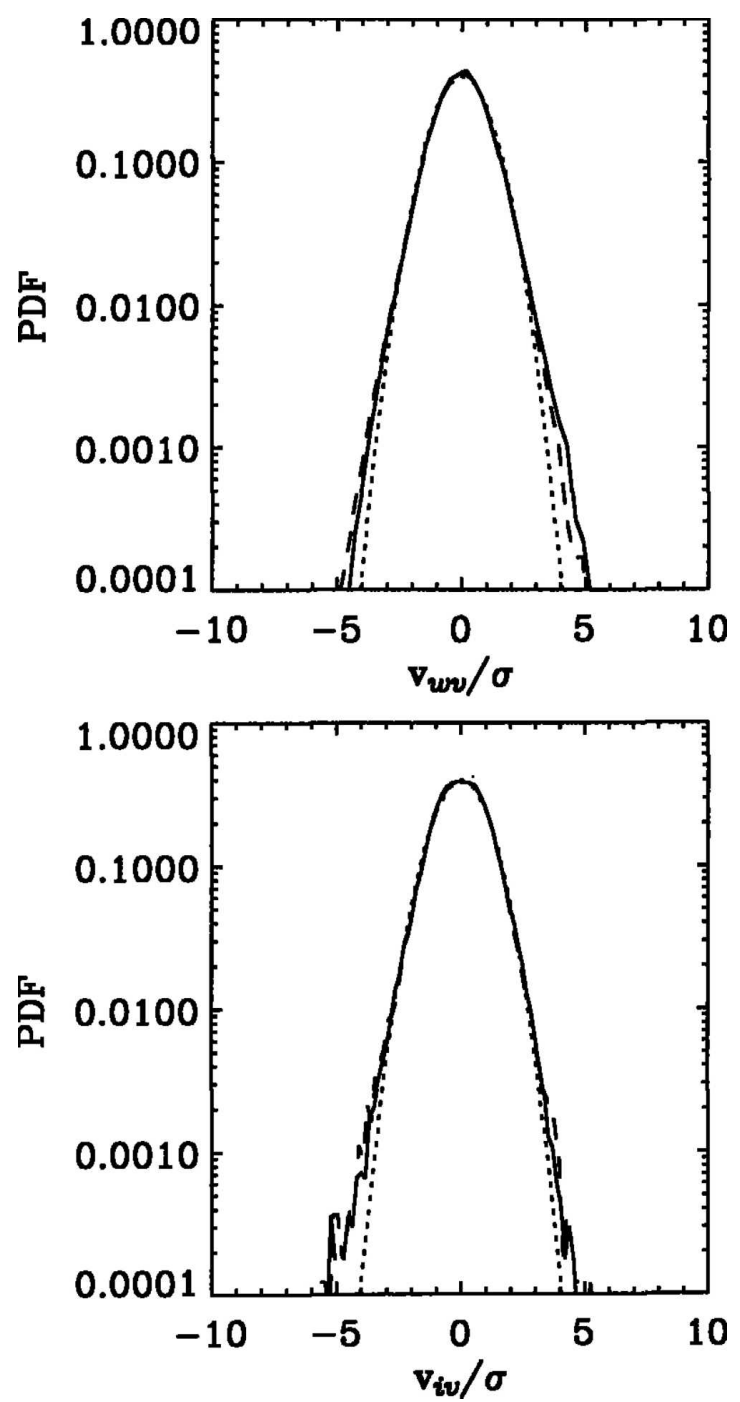

FIG. 4. Velocity PDFs inside weak vortices $\left(\mathbf{v}_{w v}\right)$ and inside intense vortices $\left(\mathbf{v}_{i v}\right)$ for the Mediterranean Sea. The solid line is for the zonal component, the dashed line is for the meridional component, and the dotted line is a Gaussian distribution with $\sigma=1$.

$$
\mathbf{v}=\mathbf{u}_{\mathrm{bg}}+\mathbf{u}_{v}+\mathbf{u}_{r},
$$

where $\mathbf{u}_{\mathrm{bg}}+\mathbf{u}_{v}=\mathbf{u}_{2}$ and $\mathbf{u}_{r}=\mathbf{v}-\mathbf{u}_{\mathrm{bg}}-\mathbf{u}_{v}=\mathbf{u}_{1}+\mathbf{u}_{3}$. Given a two-dimensional distribution of vorticity $\boldsymbol{\omega}=$ $\omega \mathbf{k}$, the associated velocity field is calculated using the Biot-Savart law:

$$
\mathbf{u}(\mathbf{x})=\frac{1}{2 \pi} \iint \frac{\mathbf{k} \times\left(\mathbf{x}-\mathbf{x}^{\prime}\right)}{\left\|\mathbf{x}-\mathbf{x}^{\prime}\right\|^{2}} \omega\left(\mathbf{x}^{\prime}\right) d A\left(\mathbf{x}^{\prime}\right) .
$$

Then, the velocity field $\mathbf{u}_{\mathrm{bg}}$ is defined as the velocity field associated with the background distribution of vorticity $\left(\omega_{\mathrm{bg}}\right)$ given by

$$
\omega_{\mathrm{bg}}= \begin{cases}\omega & \text { if } \quad W \geq-0.2 \sigma_{W} \\ 0 & \text { if } \quad W<-0.2 \sigma_{W}\end{cases}
$$


and $\mathbf{u}_{v}$ is defined as the velocity field associated with the distribution of vorticity inside vortex cores $\left(\omega_{v}\right)$ given by

$$
\omega_{v}=\left\{\begin{array}{lll}
0 & \text { if } \quad W \geq-0.2 \sigma_{W} \\
\omega & \text { if } \quad W<-0.2 \sigma_{W} .
\end{array}\right.
$$

By construction these vorticity fields are such that $\omega=$ $\omega_{\mathrm{bg}}+\omega_{v}$. Figure 5 shows the vorticity PDF corresponding to $\omega, \omega_{\mathrm{bg}}$, and $\omega_{v}$. The distribution for $\omega$ is characterized, as is the case of the velocities, by a distribution closer to an exponential than a Gaussian distribution. On the other hand, $\omega_{\mathrm{bg}}$ has a core close to a Gaussian distribution. Notice that by construction this field contains not only the background distribution of vorticity but also the distribution of vorticity associated with the surrounding cells around the vortex core (characterized by $W>0$ ). If this contribution is subtracted from the background field, the kurtosis decreases from 1.15 to 0.60 . Last, the distribution of $\omega_{v}$ lacks small values due to the capture of regions with net vorticity by the criterion $W>0.2 \sigma_{W}$.

The velocity PDFs associated with the fields $\mathbf{u}_{\mathrm{bg}}$ and $\mathbf{u}_{v}$ are represented in Fig. 6 and their first moments in Table 1. PDFs of the velocity field induced by the background distribution of vorticity $\left(\mathbf{u}_{\mathrm{bg}}\right)$ are characterized by a wide Gaussian-like core with small tails when compared with the other distributions. Its kurtosis is about a factor of 4 smaller than $\mathbf{u}_{v}$ but its skewness is the highest. The kurtosis of the background field is reduced significantly when compared with the initial velocity field (from 2.37-2.69 to 0.74-0.72), while the kurtosis of the vortex-induced velocity field is rather high for both components (2.97-3.21), which is clearly non-Gaussian, being closer to an exponential shape even at the core.

Although the PDF of the background field is closer to a Gaussian distribution, it is still significantly different from it (see section 2c). To explore this point further, the spatial distribution of the eddy kinetic energy associated with the background field, $E_{\mathrm{bg}}$, has been computed (Fig. 7). As can be seen, the $E_{\mathrm{bg}}$ is not homogeneously distributed through the basin and the highest values of $E_{\mathrm{bg}}$ are well correlated with regions characterized by a high density of coherent structures (cf. with Fig. 1). A possible explanation for this is that the definition of the background field contains both the vorticity of the background, characterized by small values of $W$ (usually taken as $|W| \leq 0.2 \sigma_{W}$ ), and the vorticity of cells that surround vortex cores (approximately

FIG. 5. Vorticity PDFs of the original field $(\omega)$, outside vortices $\left(\omega_{\mathrm{bg}}\right)$, and inside vortices $\left(\omega_{v}\right)$ for the Mediterranean Sea. The dotted line is a Gaussian distribution with $\sigma=1$.
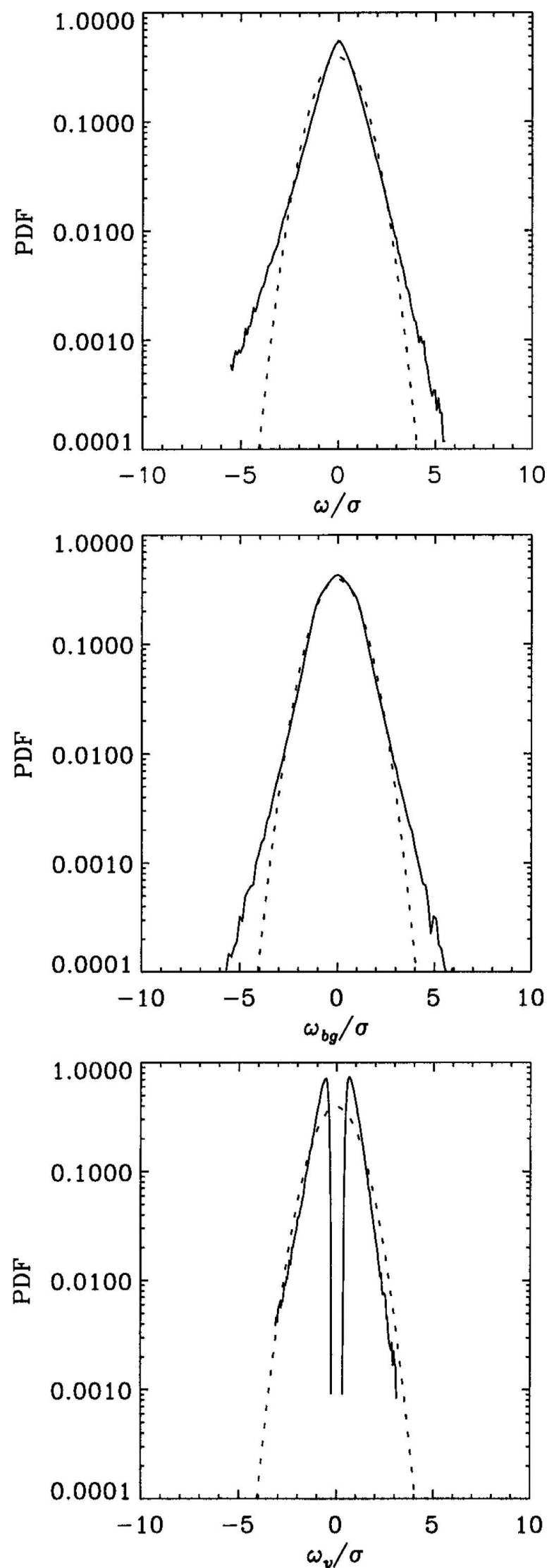

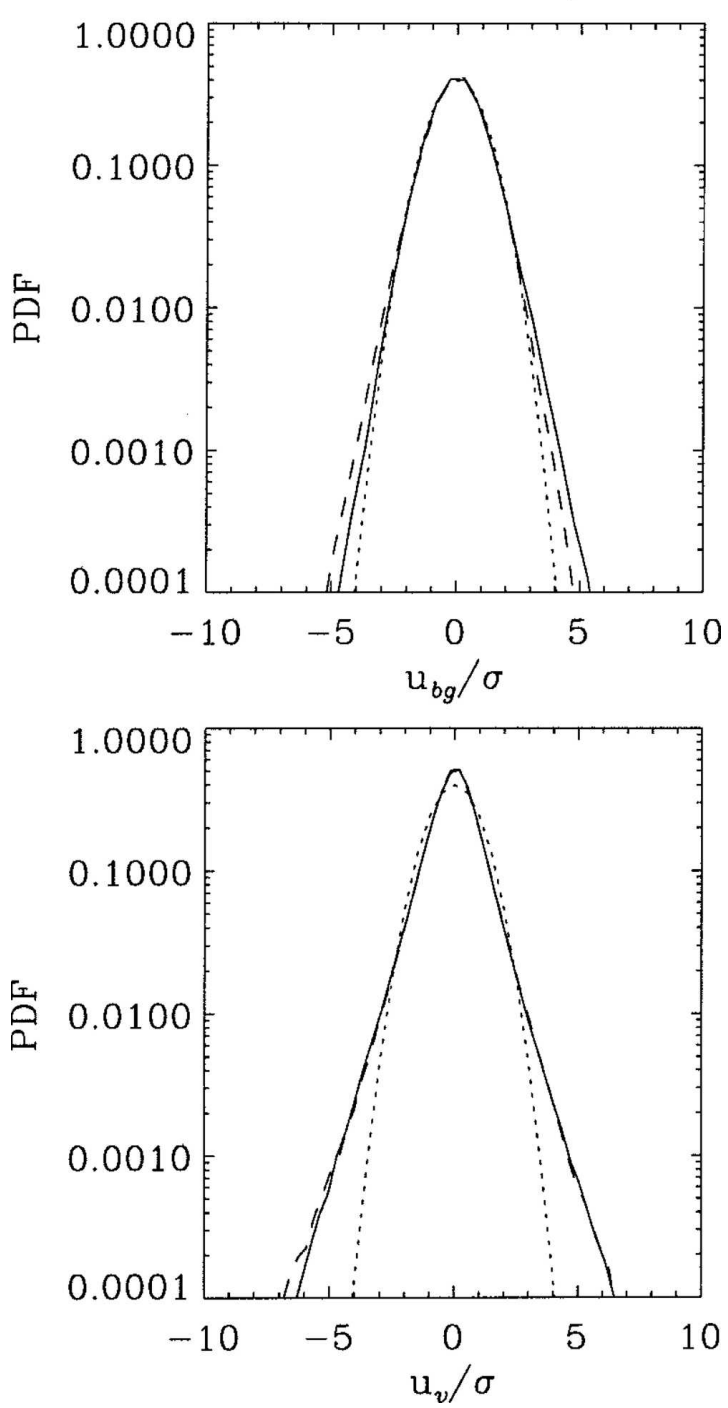

FIG. 6. Velocity PDFs of the field induced by the vorticity outside vortices $\left(\mathbf{u}_{\mathrm{bg}}\right)$ and inside vortices $\left(\mathbf{u}_{v}\right)$. The solid line is for the zonal component, the dashed line is for the meridional component, and the dotted line is a Gaussian distribution with $\sigma=1$.

taken as $\left.W>0.2 \sigma_{W}\right)$. If this last contribution is subtracted, the kurtosis of the resulting background velocity field is still reduced (from $0.74-0.72$ to $0.64-0.55$ ) but not completely eliminated. Although the vortex identification based on the Okubo-Weiss parameter is a robust criterion for the identification of vortex cores in altimetric data (see Isern-Fontanet et al. 2003; Morrow et al. 2004; Isern-Fontanet et al. 2006), it is very difficult to properly identify the surrounding cell of the vortices and therefore eliminate its contribution.

\section{c. Coherent vortex separation}

In a previous study (see Isern-Fontanet et al. 2006), it has been shown that the flow derived from SLA maps contains vortices with different energies and sizes, which leads to a rough classification of vortices into two groups: weak vortices $\left(a<2 \sigma_{W}\right)$ and intense vortices $\left(a \geq 2 \sigma_{W}\right)$. To study the contribution of each group of vortices to the PDFs, the vorticity field $\omega_{v}$ [defined in (14)] has been split into two fields: the field inside weak vortices

$$
\omega_{w v}= \begin{cases}0 & \text { if } \quad W \geq-0.2 \sigma_{W} \\ \omega & \text { inside weak vortices }\left(a<2 \sigma_{W}\right) \\ 0 & \text { inside intense vortices }\left(a \geq 2 \sigma_{W}\right)\end{cases}
$$

and the field inside intense vortices

$$
\omega_{i v}= \begin{cases}0 & \text { if } \quad W \geq-0.2 \sigma_{W} \\ 0 & \text { inside weak vortices }\left(a<2 \sigma_{W}\right) \\ \omega & \text { inside intense vortices }\left(a \geq 2 \sigma_{W}\right) .\end{cases}
$$

Again, by construction $\omega_{v}=\omega_{i v}+\omega_{w v}$. After that, these vorticity fields are integrated in order to recover $\mathbf{u}_{w v}$ and $\mathbf{u}_{i v}$ as before.

The separation of the contribution of each type of vortex leads to two distributions with different shapes and different kurtosis (see Table 1 and Fig. 8). The distributions of the velocity field generated by weak vortices has a more or less Gaussian core while their tails are relatively small in comparison with the PDF of the velocities induced by intense vortices, which looks like an exponential distribution. This suggests that the main contribution to non-Gaussianity is mainly due to intense vortices. Indeed, $\mathbf{u}_{i v}$ has the highest kurtosis of all velocity fields, while the velocity field obtained by the elimination of the contribution of intense vortices $\left(\mathbf{v}-\mathbf{u}_{i v}\right)$ is almost Gaussian, having the smallest kurtosis of the fields obtained by integrating the vorticity (see Fig. 9).

\section{Discussion and conclusions}

Results indicate that geostrophic velocity PDFs derived from SLA maps for the Mediterranean basin are significantly non-Gaussian. After the application of a flow partition based on the Okubo-Weiss parameter, this non-Gaussianity appears to be mostly due to the presence of coherent vortices, while the velocity PDF associated with the background is quite close to a Gaussian distribution. The extraction of the velocity field associated with the vortices has significantly reduced kurtosis. Indeed, similar results are obtained if only the contribution of intense vortices, which represent only about $20 \%$ of the vortices identified in the dataset, is removed from velocities. These vortices are those with amplitude values such that $a \geq 2 \sigma_{W}$ and correspond to what are generically called mesoscale vortices 


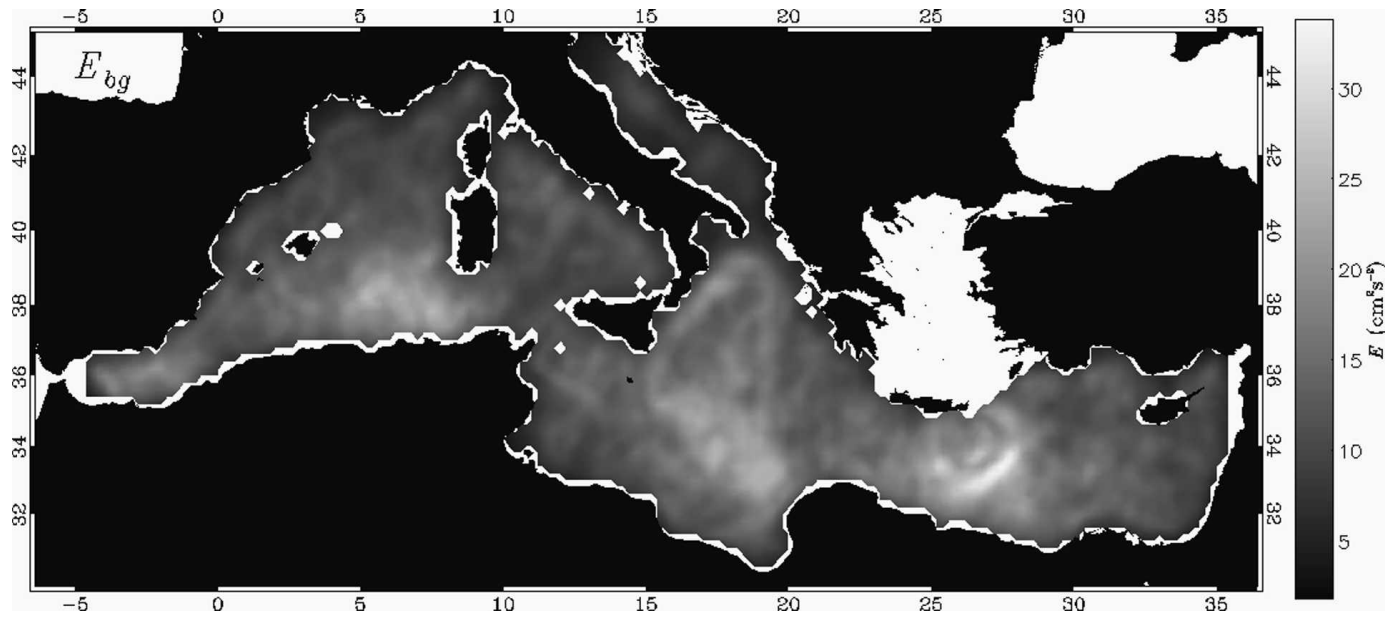

FIG. 7. Eddy kinetic energy associated with the field $\mathbf{u}_{\mathrm{bg}}\left(E_{\mathrm{bg}}\right)$

(although they are larger than the mesoscale) in the common literature on the Mediterranean.

Nevertheless, the separation between a background field with a Gaussian velocity PDF and a non-Gaussian velocity field induced by vortices is not complete. PDFs of the background field still contain small deviations from the Gaussian in their tails. As previously outlined, one reason may be due to the way the Okubo-Weiss criterion separates the field, which does not allow for extraction of the surrounding cells of vortex cores. Indeed, several studies have underlined its limitations when the flow is rapidly evolving (Basdevant and Philipovitch 1994; Hua and Klein 1998). Another reason, as proposed by Llewellyn Smith and Gille (1998), may be large-scale inhomogeneities of the EKE. The EKE in the Mediterranean was found to be inhomogeneously distributed (see Fig. 11 in Isern-Fontanet et al. 2006). Although the spatial distribution of EKE associated with the background field (Fig. 7) is much more uniform and in some regions the vortex contribution is effectively removed (i.e., the area between the Balearic Islands and the Spanish coast around $40^{\circ} \mathrm{N}, 2^{\circ} \mathrm{E}$ ), a certain degree of inhomogeneity is still apparent. In much of the domain the inhomogeneity of the EKE associated with the background field is highly correlated with regions where there is a great concentration of coherent vortices. For example, high values of EKE in the Algerian Basin, the central Ionian Sea or the Crete Passage could be explained by the contribution of cells surrounding the vortex. But a closer look into the EKE distribution for the background also reveals some patterns or traces that are probably associated with the presence of ocean currents. Beside that main currents do not appear on SLA maps, some traces of their locations and paths are not totally absent because of their associated variability, although they are not comparable with the strong signals of coherent vortices. This leads one to conclude that the origin of the nonGaussianity of the velocity field in the basin is mostly due to vortices, although some less important contributions from a different origin cannot be discounted.

A question that arises from these results is to what extent the vortices influence the dynamics of the flow in the entire basin. Our results seem to confirm that intense vortices play a major role in the dynamics of the basin. However, more refined analysis needs to be done, in particular to improve the field separation between vortices and the rest of the flow. As argued by Farge et al. (1999), methods as the one applied here do not preserve the smoothness of the vorticity field. Therefore, spurious discontinuities may contaminate the components in which the flow is split. Turiel et al. (2005, manuscript submitted to J. Atmos. Oceanic Technol., hereinafter TIG) have recently analyzed the vorticity field for the same dataset but following a completely different technique to separate the flow based on wavelets, introduced by Farge et al.: coherent vortices are extracted by wavelets projecting the vorticity, and then the field is reconstructed, discarding part of the wavelet coefficients. The velocity field associated with this coherent part, which is built from a relatively small number of wavelet coefficients, accounts for most of the enstrophy and energy of the original field (TIG). This reinforces the idea that this small percentage of intense vortices responsible for the non-Gaussian character of the velocity PDF dominate the dynamics in the Mediterranean Sea.

Another question to be studied is the role of the vertical structure in the nonlocal contribution of vortices. A 2D vortex, or an equivalent barotropic vortex, 

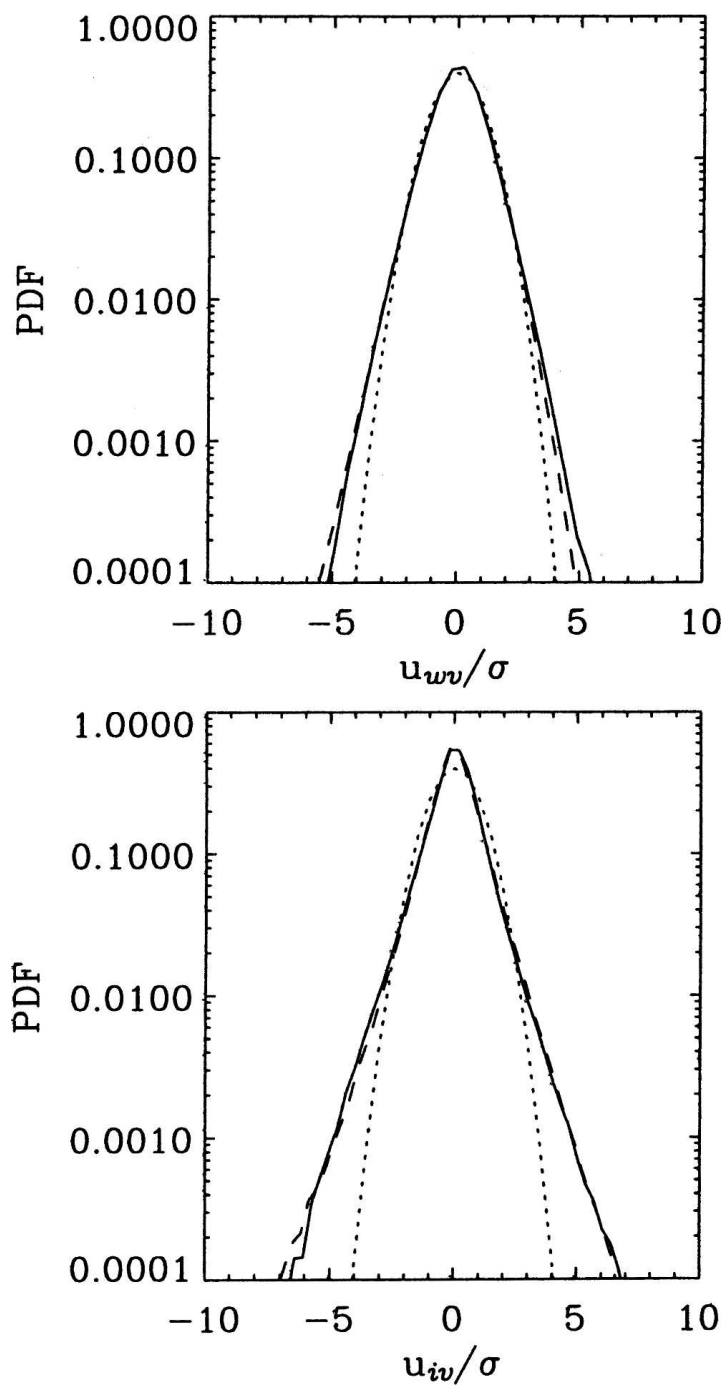

FIG. 8. Velocity PDFs of the field induced by the vorticity inside weak vortices $\left(\mathbf{u}_{w v}\right)$ and inside intense vortices $\left(\mathbf{u}_{i v}\right)$ for the Mediterranean Sea. The solid line is for the zonal component, the dashed line is for the meridional component, and the dotted line is a Gaussian distribution with $\sigma=1$.

has a larger range of influence than a baroclinic one (Bracco et al. 2004). Thus, its nonlocal effect could be different. Unfortunately, the data used in this study do not allow us to recover this information, nor is the vertical structure of coherent vortices presently known with enough detail. However, some field measurements have shown that at least some vortices in the Algerian Basin, here labeled as intense, have a very deep signature and extend down to the bottom of the Mediterranean (Millot 1985; Ruiz et al. 2002). Such vortices follow preferential paths that closely map the large-scale barotropic circulation at deep and intermediate levels, clearly constrained by $f / H$ isocontours, with $f$ being the planetary vorticity and $H$ the water depth (Testor et al.

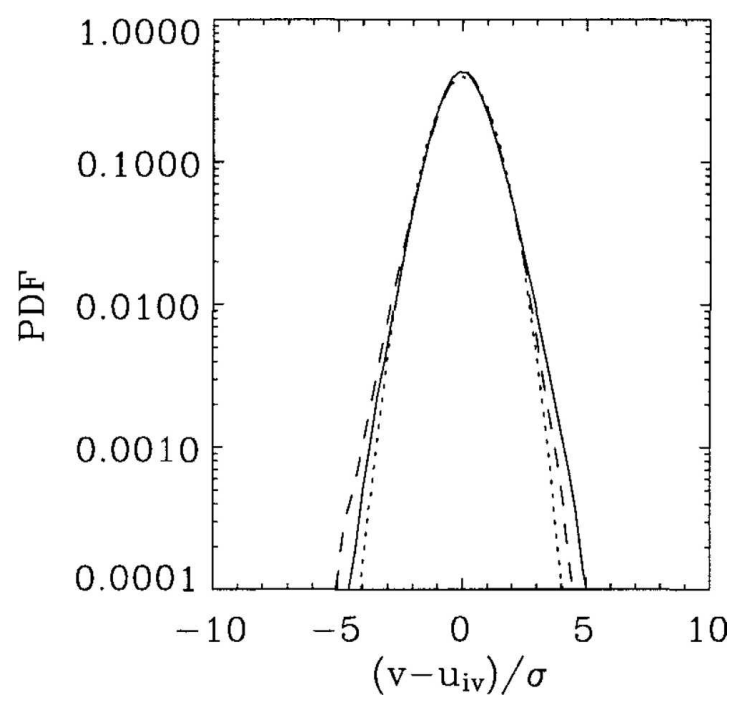

FIG. 9. Velocity PDF of the field $\mathbf{v}-\mathbf{u}_{i v}$. The solid line is for the zonal component, the dashed line is for the meridional component, and the dotted line is a Gaussian distribution with $\sigma=1$.

2005; Isern-Fontanet et al. 2006). Thus, it is expected that such marked barotropic character leads to a stronger nonlocal contribution than the one from a baroclinic vortex.

Last, a direct consequence of our work concerns the quantification of mixing and dispersion processes in the Mediterranean Sea. Non-Gaussian velocity PDFs induce anomalous dispersion due to the presence of these coherent vortices, and classical constant eddy diffusivity approaches should be discarded in favor of more complex ways of parameterizing the dispersion. To account for the anomalous dispersion due to vortices, Lagrangian models for particle dispersion may be formulated through a two-component stochastic process that separates the dynamical component associated with the background-induced motion and the vortexinduced dynamics (Thompson 1987; Pasquero et al. 2001). In such cases, the vortex-induced contribution part can be linked to the characteristics of the nonGaussian Eulerian velocity PDF through a FokkerPlanck equation. However, this point is beyond the scope of present work and is left for future studies.

Acknowledgments. This is a contribution to the IMAGEN project funded by the Spanish $\mathrm{R}+\mathrm{D}$ Plan (REN2001-0802-C02-02) and MERSEA project funded by the European Union (AIP3-CT-2003-502885). Altimetric maps for the period analyzed were elaborated upon and provided by CLS (Toulouse, France) under contract to the MATER project funded by the European Commission (MAS3-CT96-0051). 


\section{REFERENCES}

Ayoub, N., P. Le Traon, and P. De Mey, 1998: A description of the Mediterranean surface variable circulation from combined ERS-1 and TOPEX/Poseidon altimetric data. J. Mar. Syst., 18, 3-40.

Babiano, A., C. Basdevant, P. LeRoy, and R. Sadournay, 1987: Single-particle dispersion, Lagrangian structure function and Lagrangian energy spectrum in two-dimensional incompressible turbulence. J. Mar. Res., 45, 107-131.

Basdevant, C., and T. Philipovitch, 1994: On the validity of the "Weiss criterion" in two-dimensional turbulence. Physica D, 113, 17-30.

Batchelor, G. K., 1967: An Introduction to Fluid Dynamics. Cambridge University Press, 615 pp.

Bendat, J., and J. Piersol, 1985: Random Data: Analysis and Measurement Procedures. John Wiley and Sons, $691 \mathrm{pp}$.

Bracco, A., J. H. LaCasce, C. Pasquero, and A. Provenzale, 2000a: The velocity distribution of barotropic turbulence. Phys. Fluids, 12, 2478-2488.

_ J. LaCasce, and A. Provenzalle, 2000b: Velocity probability density functions for oceanic floats. J. Phys. Oceanogr., 30, 461-474.

- E. Chassignet, Z. Garraffo, and A. Provenzalle, 2003: Lagrangian velocity distributions in high-resolution numerical simulations of the North Atlantic. J. Atmos. Oceanic Technol., 20, 1212-1220.

_ J. Jon Hardenberg, A. Provenzalle, J. B. Weiss, and J. C. McWilliams, 2004: Dispersion and mixing in quasigeostrophic turbulence. Phys. Rev. Lett., 92, 084501, doi:10.1103/ PhysRevLett.92.084501.

CLS, 1996: Corrected sea surface heights products: AVISO user handbook. 2.0 ed. AVI-NT-011-311-CN, Collecte Localisation Satellites, Ramonville, France, 21 pp.

Emery, W. J., and R. E. Thomson, 1998: Data Analysis Methods in Physical Oceanography. Pergamon, 634 pp.

Farge, M., K. Schneider, and N. Kevlahan, 1999: Non-Gaussianity and coherent vortex simulation for two-dimensional turbulence using an adaptive orthogonal wavelet basis. Phys. Fluids, 11, 2187-2201.

Font, J., J. Isern-Fontanet, and J. Salas, 2004: Tracking a big anticyclonic eddy in the Algerian basin (western Mediterranean Sea). Sci. Mar., 68, 331-342.

Gille, S. T., and S. G. Llewellyn Smith, 2000: Velocity probability density functions from altimetry. J. Phys. Oceanogr., 30, 125136.

Hua, B. L., and P. Klein, 1998: An exact criterion for the stirring properties of nearly two-dimensional turbulence. Physica D, 113, 98-110.

Isern-Fontanet, J., E. García-Ladona, and J. Font, 2003: Identification of marine eddies from altimetry. J. Atmos. Oceanic Technol., 20, 772-778.

$\longrightarrow, \ldots$, and $\longrightarrow, 2006$ : The vortices of the Mediterranean Sea: An altimetric viewpoint. J. Phys. Oceanogr., 36, 87-103.

Jarque, C., and A. Bera, 1981: Efficient tests for normality, homoscedasticity and serial independence of regression residuals. Econ. Lett., 7, 313-318.

Jiménez, J., 1996: Algebraic probability density tails in decaying isotropic two-dimensional turbulence. J. Fluid Mech., 313, 223-240.
LaCasce, J. H., 2005: On the Eulerian and Lagrangian velocity distributions in the North Atlantic. J. Phys. Oceanogr., 35, 2327-2336.

Larnicol, G., P. Le Traon, N. Ayoub, and P. De Mey, 1995: Mean sea level and surface circulation variability of the Mediterranean Sea from 2 years of TOPEX/Poseidon altimetry. J. Geophys. Res., 100, 385-396.

— , N. Ayoub, and P. Le Traon, 2002: Major changes in the Mediterranean sea level variability from 7 years of TOPEX/ Poseidon ERS-1/2 data. J. Mar. Syst., 33-34, 63-89.

Le Traon, P., and F. Ogor, 1998: ERS-1/2 orbit improvement using TOPEX/Poseidon: The $2 \mathrm{~cm}$ challenge. J. Geophys. Res., 103, 8045-8057.

_ - F. Nadal, and N. Ducet, 1998: An improved mapping method of multisatellite altimeter data. J. Atmos. Oceanic Technol., 15, 522-534.

Llewellyn Smith, S. G., and S. T. Gille, 1998: Probability density functions of large-scale turbulence in the ocean. Phys. Rev. Lett., 81, 5249-5252.

Matteoda, A., and S. M. Glenn, 1996: Observation of recurrent mesoscale eddies in the eastern Mediterranean. J. Geophys. Res., 101, 20 687-20 709.

Maurizi, A., A. Griffa, P. Poulian, and F. Tampieri, 2004: Lagrangian turbulence in the Adriatic Sea as computed from drifter data: Effects of inhomogeneity and nonstationarity. $J$. Geophys. Res., 109, C04010, doi:10.1029/2003JC002119.

McWilliams, J. C., 1984: The emergence of isolated coherent vortices in turbulent flow. J. Fluid Mech., 146, 21-43.

- 1990: The vortices of two-dimensional turbulence. J. Fluid Mech., 219, 361-385.

Millot, C., 1985: Some features of the Algerian Current. J. Geophys. Res., 90, 7169-7176.

_ 1999: Circulation in the western Mediterranean Sea. J. Mar. Syst., 20, 423-442.

_ 2005: Circulation in the Mediterranean Sea: Evidences, debates and unanswered questions. Sci. Mar., 69 (Suppl. 1), $5-21$.

Morrow, R., F. Birol, D. Griffin, and J. Sudre, 2004: Divergent pathways of cyclonic and anti-cyclonic ocean eddies. Geophys. Res. Lett., 31, L24311, doi:10.1029/2004GL020974.

Okubo, A., 1970: Horizontal dispersion of floatable particles in the vicinity of velocity singularities such as convergences. Deep-Sea Res., 17, 445-454.

Pasquero, C., A. Provenzale, and A. Babiano, 2001: Parametrization of dispersion in two-dimensional turbulence. J. Fluid Mech., 439, 279-303.

Press, W. H., S. A. Teukolsky, W. T. Vetterling, and B. P. Flannery, 1994: Numerical Recipes in FORTRAN: The Art of Scientific Computing. Cambridge University Press, 933 pp.

Provenzale, A., 1999: Transport by coherent barotropic vortices. Annu. Rev. Fluid Mech., 31, 55-93.

Puillat, I., I. Taupier-Letage, and C. Millot, 2002: Algerian eddies lifetime can near 3 years. J. Mar. Syst., 31, 245-259.

Rhines, P. B., 1979: Geostrophic turbulence. Annu. Rev. Fluid Mech., 11, 401-441.

Ruiz, S., J. Font, M. Emelianov, J. Isern-Fontanet, C. Millot, J. Salas, and I. Taupier-Letage, 2002: Deep structure of an open sea eddy in the Algerian Basin. J. Mar. Syst., 33-34, 179-195.

Salas, J., E. García-Ladona, and J. Font, 2001: Statistical analysis of the surface circulation in the Algerian Current using Lagrangian buoys. J. Mar. Syst., 29, 69-85.

_ _ C. Millot, J. Font, and E. García-Ladona, 2002: Analysis of 
mesoscale phenomena in the Algerian Basin from drifting buoys and infrared images. Deep-Sea Res., 49, 245-266.

Schorghofer, N., and S. T. Gille, 2002: Statistics of velocity gradients in two-dimensional Navier-Stokes and ocean turbulence. Phys. Rev. E, 65, 026307, doi:10.1103/PhysRevE.65.026307.

Swenson, M. S., and P. P. Niiler, 1996: Statistical analysis of the surface circulation. J. Geophys. Res., 101, 22 631-22 645.

Testor, P., U. Send, J. Gascard, C. Millot, I. Taupier Letage, and K. Béranger, 2005: The mean circulation of the southwestern
Mediterranean Sea: Algerian gyres. J. Geophys. Res., 110, C11017, doi:10.1029/2004JC002861.

Thompson, D. J., 1987: Criteria for the selection of stochastic models of particle trajectories in turbulent flows. J. Fluid Mech., 180, 529-556.

Weiss, J. B., 1991: The dynamics of enstrophy transfer in two-dimensional hydrodynamics. Physica D, 48, 273-294.

_, and J. C. McWilliams, 1993: Temporal scaling behavior of decaying two-dimensional turbulence. Phys. Fluids A, 5, 608 621. 